\title{
Rinoplastía y rinoseptoplastía funcional: Mejorando la función nasal más allá de la cirugía del tabique y los cornetes nasales
}

\section{Functional rhinoplasty and septorhinoplasty: Improving nasal function beyond septal and turbinate surgery}

\author{
Andrés Alvo $V^{1,2}$
}

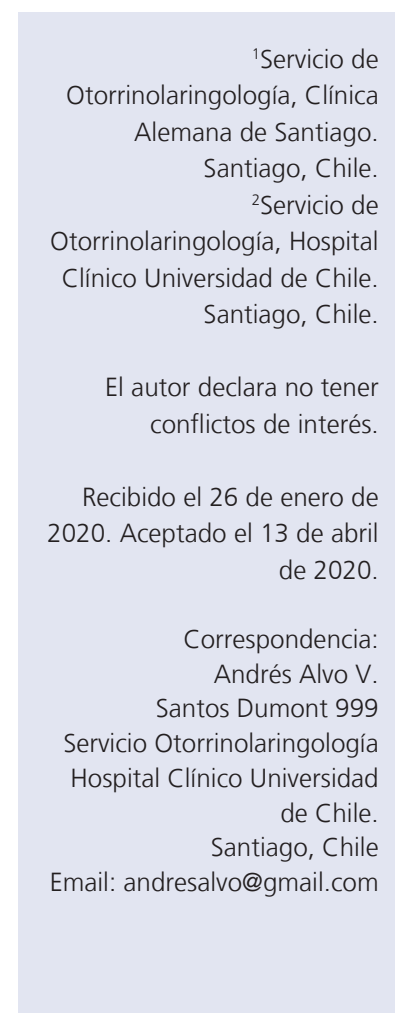

\section{Resumen}

La obstrucción nasal es una de las causas más frecuentes de consulta otorrinolaringológica general. Diversas estructuras juegan un rol en la mantención de una adecuada función respiratoria nasal, incluyendo el tabique, los cornetes, las paredes laterales y las alas nasales. La cirugía del tabique y de los cornetes inferiores son los procedimientos más comúnmente realizados y aunque son efectivos en un gran porcentaje de los casos, en ocasiones se deben intervenir otras estructuras nasales para corregir adecuadamente la obstrucción. La cirugía con fines primariamente funcionales de las válvulas nasales externa e interna, de la punta y/o de la pirámide ósea se ha denominado "rinoplastía funcional". Dada la importancia de estas estructuras para la recuperación de una adecuada permeabilidad nasal estática y dinámica, el diagnóstico y manejo quirúrgico de estas condiciones debe ser de dominio del otorrinolaringólogo.

Palaras clave: obstrucción nasal, válvula nasal, rinoplastía, septoplastía, turbinoplastía.

\begin{abstract}
Nasal obstruction is one of the most frequent complaints in general otorhinolaryngology practice. Different structures have a role in maintaining an adequate nasal breathing function, including the septum, turbinates, lateral sidewalls and nasal alae. Surgery of the nasal septum and inferior turbinates are the most commonly performed procedures and, although effective in most cases, occasionally other nasal structures must be intervened to correctly address the sites of obstruction. The term "functional rhinoplasty" has been coined for surgery of the external and internal nasal valves, nasal tip and/or bony pyramid, with primarily functional objectives. Given the importance of these structures for restoring an adequate static and dynamic nasal patency, diagnosis and management of these conditions must be dominion of the otorhinolaryngologist.
\end{abstract}

Keywords: nasal obstruction, nasal valve, rhinoplasty, septoplasty, turbinoplasty.

\section{Introducción}

La obstrucción nasal es una de las causas más frecuentes de consulta otorrinolaringológica general. Sus etiologías son múltiples, incluyendo factores anatómicos, inflamación/ edema, secreciones, malformaciones, tumores y cuerpos extraños, entre otros ${ }^{1}$. Respecto a las causas anatómicas, diversas estructuras juegan un rol en la mantención de una ade- cuada función respiratoria nasal, incluyendo el tabique, los cornetes, las paredes laterales y las alas nasales. Se estima que las fosas nasales serían responsables de al menos el 50\% de la resistencia total de la vía aérea ${ }^{2}$. Las estructuras antes mencionadas conforman zonas de mayor resistencia denominadas válvulas nasales. La válvula nasal externa (VNE) es un espacio volumétrico ubicado entre la narina caudalmente, el tabique y la crus media del cartílago 
alar (CA) hacia medial, el CA y tejido fibrograso anterolateralmente, y la apertura hacia la válvula interna hacia posterior ${ }^{3}$, mientras que la espina nasal y el piso de la fosa nasal conforman su límite inferior ${ }^{4}$. Por otro lado, la válvula nasal interna (VNI) es un área comprendida entre el septum nasal medialmente, el borde caudal del cartílago lateral superior (CLS) superolateralmente, la cabeza del cornete inferior inferomedialmente y el piso nasal inferiormente ${ }^{4}$ (Figura 1).

Se considera que la VNI es la zona más estrecha de la fosa nasal y que el ángulo entre el CLS y el tabique es de 9 a 15 grados en población caucásica ${ }^{5}$. La interacción entre estas distintas estructuras puede condicionar que la obstrucción sea estática, dinámica o mixta. Además, distintas etiologías pueden y suelen combinarse en un mismo paciente, haciendo necesario un diagnóstico preciso para poder plantear el mejor plan de tratamiento. Los procedimientos quirúrgicos más comúnmente realizados para el tratamiento de la obstrucción nasal en adultos son la septoplastía y las distintas cirugías orientadas a disminuir el tamaño de los cornetes inferiores. Aunque altamente efectivos en un gran porcentaje de los casos, en ocasiones se requiere actuar sobre otras estructuras de la nariz para restaurar un adecuado flujo aéreo nasal.

\section{Evaluación clínica y estudio de la obstrucción nasal de origen anatómico-funcional}

Después de obtener una historia clínica meticulosa, un adecuado examen físico será el aspecto más importante en el diagnóstico de estos pacientes. La inspección externa detectará laterorrinias, hundimientos óseos, ptosis y algunos colapsos valvulares, mientras que la evaluación endonasal mediante rinoscopía anterior permitirá observar desviaciones del tabique nasal e hipertrofia de los cornetes nasales, además de evidenciar otras patologías cicatriciales, inflamatorias o tumorales.

Funcionalmente, se deben valorar las válvulas nasales, lo que se puede hacer mediante inspiración forzada o con la maniobra de Cottle. Esta consiste en traccionar lateralmente la mejilla, lo que tensa la pared lateral nasal y abre la VNI. La prueba se considera positiva cuando el flujo aéreo inspiratorio por la fosa

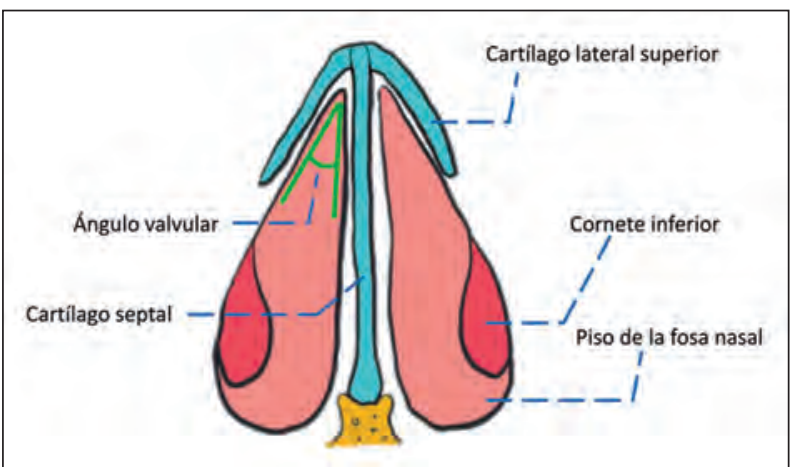

Figura 1. Componentes de la válvula nasal interna.

examinada mejora, lo que se observa en pacientes con válvulas estrechas o colapsadas ${ }^{6}$. Aunque muchas personas notarán cierto beneficio en el flujo nasal, en casos de colapso esta mejoría será sustancial. Otra forma de evaluar la VNI consiste en levantar y soportar el ángulo valvular interno introduciendo algún instrumento dentro de la fosa nasal, como una cureta de oído ${ }^{7}$.

Con el fin de cuantificar los síntomas y poder hacer comparaciones entre pacientes o entre tratamientos, se han desarrollado cuestionarios específicos para la obstrucción nasal. La más utilizada es la NOSE, que da valores del 0 al 4 a los síntomas nasales de congestión, obstrucción, dificultad respiratoria, dificultad para dormir y dificultad para ejercitarse ${ }^{8}$. En general, estas escalas tienen mayor relevancia en investigación que en la práctica clínica habitual. En ocasiones, los hallazgos clínicos pueden ser complementados con endoscopía (nasofibroscopía y endoscopía nasal rígida), tomografía computarizada de nariz y cavidades paranasales, rinomanometría y/o rinometría acústica. Los estudios endoscópicos y por imágenes podrían detectar algunas causas de obstrucción nasal no evidenciadas durante la rinoscopia anterior, por lo que tienen un rol importante en caso de que el examen físico no explique completamente los síntomas o ante duda diagnóstica. Por otra parte, la rinomanometría y la rinometría acústica son exámenes que buscan medir la obstrucción nasal de manera objetiva. La primera es una prueba funcional que mide flujo aéreo y presión transnasal mediante transductores de presión, mientras que la segunda utiliza ondas de so- 
nido para calcular áreas seccionales a distintas profundidades de la fosa nasal. Estos últimos dos exámenes son frecuentemente solicitados por seguros médicos, pero tienen limitaciones para evaluar obstrucción por ptosis de la punta y por colapso alar, poseen problemas de variabilidad operador-dependiente y de correlación con los síntomas del paciente ${ }^{1}$.

\section{Cirugía del tabique nasal}

La septoplastía consiste en el abordaje del tabique cartilaginoso y óseo a través de un túnel subpericóndrico-subperióstico, con remodelación y/o resección de los segmentos desviados, manteniendo o reconstruyendo siempre una adecuada estructura de soporte para el dorso y la punta nasal. Generalmente se acepta que la preservación de un marco de tabique cartilaginoso dorsal y caudal (" $L$ strut") de al menos 1 a $1,5 \mathrm{~cm}$ es suficiente para este propósito. Este marco se relaciona con los huesos nasales hacia cefálico en la llamada "keystone area" y hacia inferior y caudal con la espina nasal anterior (Figura 2).

En la cirugía es también importante identificar y tratar obstrucciones debido a prominencias o desviaciones de la cresta maxilar', estructura donde se apoya inferiormente el tabique nasal. Aunque en la mayoría de los casos, la septoplastía se realiza mediante incisiones endonasales, en ocasiones es necesario acceder mediante incisiones externas (septoplastía abierta) ${ }^{10}$ o bien extraer el tabique cartilagi-

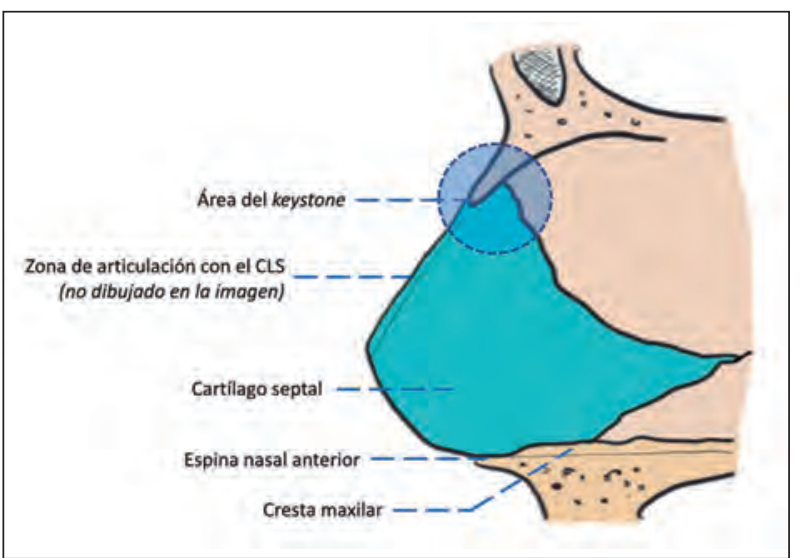

Figura 2. Área del keystone o piedra clave: Zona del dorso donde los huesos nasales se encuentran con los cartílagos laterales superiores y el cartílago septal. noso, remodelarlo y luego reposicionarlo en el paciente (septoplastía extracorpórea) ${ }^{11}$. Esto puede estar especialmente indicado en caso de desviaciones septales altas, necesidad de uso de injertos espaciadores o de extensión septal, tabiques severamente desviados o con depleción importante de cartílago. Muchos cirujanos favorecen también el uso de endoscopios, que permiten identificar mejor los planos, abordar de manera mínimamente invasiva espolones septales, acceder a segmentos más altos del tabique, poder ver y mostrar el procedimiento en una pantalla, entre otras ventajas ${ }^{12}$. Otra condición particular en la cirugía del tabique nasal son las desviaciones del septum caudal ${ }^{13}$, que pueden requerir de técnicas de remodelación especiales para preservar o reconstruir el marco dorso-caudal que proporciona parte importante del soporte de la punta y del dorso nasal.

\section{Cirugía de los cornetes inferiores}

Los cornetes nasales son estructuras de la pared lateral de la fosa nasal. Los más constantes son el superior, medio e inferior; y de éstos, el inferior es el único formado por un hueso distinto (los otros dependen del hueso etmoidal). El cornete inferior está compuesto por un centro óseo, una capa submucosa con glándulas y una mucosa epitelial. Su estructura plegada puede aumentar la superficie mucosa endonasal hasta $200 \mathrm{~cm}^{2}$ y contribuye en varios aspectos de la función nasal, como la humidificación y termorregulación del aire inspirado, la regulación del flujo nasal y la producción de secreciones. Para esto consta de un tejido eréctil ricamente vascularizado y una compleja regulación autonómica ${ }^{14,15}$.

El manejo de la obstrucción nasal por cornetes inferiores aumentados de tamaño incluye tanto tratamientos médicos como quirúrgicos. Mientras que los medicamentos actúan únicamente en los tejidos blandos, la cirugía puede actuar sobre éstos, la estructura ósea o ambos. Las cirugías incluyen la lateralización o outfracture; resección submucosa del hueso conchal; resección submucosa con microdebridador; reducción submucosa con radiofrecuencia, electrocauterización u otros; y la turbinectomía parcial ${ }^{15,16}$. En general, las turbinectomías totales son poco utilizadas para esta indicación debido al mayor riesgo teórico 
de alterar la función nasal, rinitis atrófica y síndrome de nariz vacía ${ }^{17-20}$.

\section{Efectividad de la cirugía de tabique y cornetes en el manejo de la obstrucción nasal}

A pesar de que el tratamiento quirúrgico de la obstrucción nasal es una práctica común en Otorrinolaringología, van Egmond y cols. presentaron una revisión sistemática en 2018 en la que no lograron encontrar estudios de buena calidad que compararan la cirugía con el tratamiento no quirúrgico ${ }^{21}$. Por lo tanto, al año siguiente, el mismo grupo publicó un estudio clínico controlado aleatorizado en la que demostraron que la cirugía fue superior al manejo conservador, tanto en mejoría clínica como en flujo inspiratorio nasal ${ }^{22}$. En otro estudio del 2018 por Valsamidis y cols., la septoplastía con cauterización de cornetes demostró mejorías significativas en cuanto a síntomas y permeabilidad nasal, aunque el efecto subjetivo tendió a disminuir con el paso del tiempo postoperatorio ${ }^{23}$. En este mismo sentido, diversos autores han reportado resultados exitosos: Jenssen y cols. publicaron un $69 \%$ de satisfacción a 9 años postoperatorios ${ }^{24}$; Arunachalam y cols. una mejoría significativa en la obstrucción nasal en un 74\% de los pacientes ${ }^{25}$; y Gandomi y cols. un $89,5 \%$ en pacientes jóvenes ${ }^{26}$. Estos mismos autores reportan una tasa de éxito en la literatura de entre un $63 \%$ y $85 \%{ }^{26}$. Asimismo, Stewart y cols. presentaron el 2004 sus resultados de evaluación de cirugía septal con o sin reducción de cornetes, demostrando una disminución significativa en el puntaje NOSE de síntomas obstructivos nasales y un porcentaje de pacientes insatisfechos de sólo $6 \%$ a los 3 meses postcirugía ${ }^{27}$.

En vista de lo anterior, aunque la cirugía de tabique y cornetes ha demostrado ser efectiva en un gran porcentaje de los casos, existe un grupo de pacientes en los que no parece ser suficiente para mejorar los síntomas. En este contexto pueden existir varios factores involucrados, como un diagnóstico inadecuado de la causa de la obstrucción nasal; mala técnica quirúrgica; zonas anatómicas no intervenidas; memoria de los tejidos operados; coexistencia de patología inflamatoria no tratada; entre otros. En cuanto a los problemas derivados de la omisión quirúrgica, son comunes las des- viaciones residuales dorsocaudales, el colapso valvular no tratado y el insuficiente manejo de los cornetes nasales. Con cierta frecuencia se observan también problemas iatrogénicos como sinequias y perforaciones septales ${ }^{28,29}$.

\section{Rinoplastía funcional}

En ocasiones, la obstrucción nasal puede deberse también a problemas estructurales de la nariz externa, como las alas, punta, válvulas y la pared lateral ósea. El término "rinoplastía funcional" comprende todas las técnicas dirigidas a modificar la estructura externa con el objetivo de mejorar la respiración nasal ${ }^{30}$. Una revisión sistemática y metaanálisis reciente encontró un cambio significativo en los puntajes NOSE de síntomas obstructivos nasales después de la rinoplastia funcional a los 12 meses, con una reducción de 43-50 puntos (de un total de 100), aunque limitados por la heterogeneidad de los estudios ${ }^{30}$. El 2015, Chambers y cols. publicaron un estudio sobre el tratamiento de la obstrucción nasal mediante cirugía de la válvula nasal en pacientes con historia de septoplastía previa no exitosa, describiendo un $95 \%$ de estrechamiento moderado a severo de la VNI, $48 \%$ de colapso de la VNI, 45\% de estrechamiento de la VNE y $40 \%$ de colapso de la VNE. De manera interesante, encontraron también un 65\% de desviación septal alta. El puntaje NOSE disminuyó de 75,7 preoperatoriamente a 22,1 a los 6 meses o más postoperatoriamente ${ }^{31}$. Del punto de vista del cirujano, en una encuesta realizada a otorrinolaringólogos canadienses, el colapso valvular nasal no tratado fue la segunda causa más común de falla de la cirugía de obstrucción nasal $(25,6 \%)^{32}$.

Respecto a la VNE, se han descrito distintas técnicas para mejorar la fuerza y estabilidad de esta zona, incluyendo injertos como el de reborde alar y el listón alar, y suturas como la de tracción de crus lateral. Otras técnicas de utilidad están dirigidas a tratar una concavidad anormal de la crus lateral, como vástagos y reposiciones ${ }^{33}$. Una técnica sencilla y útil es usar la porción cefálica de la crus lateral, que normalmente se reseca, como soporte al doblarlo sobre sí mismo, maniobra conocida como "turn-in flap" 34 . En cuanto a la VNI, la importancia del adecuado manejo de la bóveda nasal media es fundamental para obtener 
buenos resultados estéticos y funcionales en rinoplastía. Los injertos espaciadores o spreader grafts se han convertido en el caballo de batalla para la reconstrucción de la VNI. De acuerdo a la ley de Poiseuille, incluso disminuciones pequeñas del ángulo normal de 10-15 grados entre el CLS y el septum pueden determinar una obstrucción significativa de la vía aérea a nivel de la VNI. Por otro lado, cualquier debilidad estructural de esta zona la hace susceptible al colapso cuando aumenta el flujo, siguiendo el principio de Bernoulli ${ }^{35}$. Los injertos espaciadores consisten en listones de cartílago que se posicionan y suturan entre los CLS y el tabique nasal, para ensanchar la VNI. Además, permiten restaurar las líneas estéticas dorsales, reconstruir el dorso nasal y ayudar a enderezar algunas laterorrinias ${ }^{36,37}$. Una modificación a esta técnica consiste en utilizar parte del mismo CLS y plegarlo sobre sí mismo para restaurar la forma en T de la bóveda cartilaginosa, maniobra llamada spreader fla ${ }^{38,39}$. Otras técnicas dirigidas a sostener o aumentar el ángulo valvular son los injertos en mariposa y las suturas ensanchadoras o flaring sutures ${ }^{33}$.

Un inadecuado soporte de la punta nasal también puede ser motivo de obstrucción, como mencionan Novoa y cols., describiendo una mejoría funcional en el $86 \%$ de los casos con su técnica "I-Beam" nasal ptósica suele ser subestimado y su corrección puede tener efectos beneficiosos sobre la obstrucción nasal. La estrategia quirúrgica dependerá de las estructuras alteradas, como un septum largo, cruras laterales largas o cruras mediales $\operatorname{cortas}^{41}$. Otro aspecto a considerar es el manejo de la pared ósea. Aunque es menos mencionada en la literatura, probablemente todos los cirujanos de nariz han tenido casos en los que una laterorrinia ósea o un hundimiento de huesos nasales impide una adecuada corrección funcional. En esta línea, Veit y cols. publicaron su experiencia en 398 rinoseptoplastías de revisión, de los cuales el 14\% tuvieron un diagnóstico de desviación nasal ósea y de la lámina perpendicular del etmoides. Todos los pacientes mejoraron su obstrucción nasal después de la cirugía de revisión ${ }^{42}$.

Todos los aspectos discutidos anteriormente pueden estar presentes no solo como una alteración congénita o traumática, sino que también después de una rinoseptoplastía pre- via, ya sea por no haber tratado adecuadamente la causa de la obstrucción, por debilitamiento estructural, por un nuevo colapso anatómico y/o por obstrucción cicatricial; estos temas han sido revisados extensamente por Joe en el 2004 ${ }^{43}$. Algunas situaciones posoperatorias comúnmente observadas son el colapso de la VNI por daño de la región del keystone y resección agresiva de los CLS, o de la VNE por una sobre-resección cefálica de los CA, dejando un marco menor a $6 \mathrm{~mm}^{44}$. Asimismo, osteotomías laterales que no respetan la zona de la apertura piriforme bajo el nivel de la inserción del cornete inferior (triángulo de Webster) pueden producir medialización ósea con compromiso del área valvular ${ }^{45}$.

\section{Conclusión}

El diagnóstico y tratamiento de la obstrucción nasal es una de las áreas principales de la práctica otorrinolaringológica. Dependiendo de la causa, un porcentaje importante de pacientes tendrán indicación de manejo médico, pero en caso de una obstrucción anatómica o pérdida de soporte estructural podrán requerir de cirugía. En este contexto, la septoplastía y la cirugía de cornetes inferiores son los procedimientos más comunes y, bien indicados, tienen una alta probabilidad de éxito. En ocasiones, la obstrucción compromete otras regiones de la nariz, como las válvulas nasales, la punta o la pirámide ósea, por lo que se requiere de técnicas que actúen también en la nariz externa. El conjunto de procedimientos quirúrgicos orientados a los tejidos blandos, cartílagos y huesos de la pirámide nasal externa han sido denominados rinoplastia funcional, en donde el objetivo primario es mejorar o restaurar una adecuada permeabilidad nasal. Aunque cambios estéticos pueden resultar de, por ejemplo, utilizar injertos espaciadores o corregir laterorrinias óseas, esta no es la razón primordial de la cirugía. Una adecuada evaluación de las posibles causas de obstrucción, seguidas de una discusión con el paciente respecto a las posibles alternativas terapéuticas y extensión de la cirugía, y un preciso tratamiento quirúrgico de las alteraciones detectadas, debe ser campo y dominio del otorrinolaringólogo general. 


\section{Bibliografía}

1. Chandra RK, Patadia MO, Raviv J. Diagnosis of nasal airway obstruction. Otolaryngol Clin North Am 2009;42:207-225.

2. McNicholas WT. The nose and OSA: variable nasal obstruction may be more important in pathophysiology than fixed obstruction. Eur Respir J 2008;32:3-8.

3. Hamilton GS 3rd. The External Nasal Valve. Facial Plast Surg Clin North Am 2017;25:179-194.

4. Friedman O, Cekic E, Gunel C. Functional Rhinoplasty. Facial Plast Surg Clin North Am 2017;25:195-199.

5. Patel B, Virk JS, Randhawa PS, Andrews PJ. The internal nasal valve: a validated grading system and operative guide. Eur Arch Otorhinolaryngol 2018; 275:2739-2744.

6. Bonaparte JP, Campbell R. A prospective cohort study assessing the clinical utility of the Cottle maneuver in nasal septal surgery. J Otolaryngol Head Neck Surg 2018;47:45.

7. Fung E, Hong P, Moore C, Taylor SM. The effectiveness of modified Cottle maneuver in predicting outcomes in functional rhinoplasty. Plast Surg Int 2014;2014:618313.

8. Stewart MG, Witsell DL, Smith TL, Weaver EM, Yueh B, Hannley MT. Development and validation of the Nasal Obstruction Symptom Evaluation (NOSE) scale. Otolaryngol Head Neck Surg 2004;130:157-163.

9. Pons Y, Champagne C, Genestier L, Ballivet de Régloix S. Endoscopic septoplasty: Tips and pearls. Eur Ann Otorhinolaryngol Head Neck Dis 2015;132:353-356.

10. Chaaban M, Shah AR. Open septoplasty: indications and treatment. Otolaryngol Clin North Am 2009;42:513-519.

11. McGrath M, Bell E, Locketz GD, Becker DG. Review and update on extracorporeal septoplasty. Curr Opin Otolaryngol Head Neck Surg 2019; 27:1-6.

12. Sautter NB, Smith TL. Endoscopic septoplasty. Otolaryngol Clin North Am 2009;42:253-260.

13. Haack J, Papel ID. Caudal septal deviation. Otolaryngol Clin North Am 2009;42:427-436.

14. Smith DH, Brook CD, Virani S, Platt MP. The inferior turbinate: An autonomic organ. Am J Otolaryngol 2018;39:771-775.

15. Downs BW. The Inferior Turbinate in Rhinoplasty. Facial Plast Surg Clin North Am 2017;25:171-177.

16. Ye T, Zhou B. Update on surgical management of adult inferior turbinate hypertrophy. Curr Opin Otolaryngol Head Neck Surg 2015;23:29-33.

17. Nurse LA, Duncavage JA. Surgery of the inferior and middle turbinates. Otolaryngol Clin North Am 2009;42:295-309.

18. Kuan EC, Suh JD, Wang MB. Empty nose syndrome. Curr Allergy Asthma Rep 2015; 15:493.
19. Payne SC. Empty nose syndrome: what are we really talking about? Otolaryngol Clin North Am 2009;42:331-337.

20. García D, Jara N, Fernández L, Naser A. Síndrome de naríz vacía. Rev Otorrinolaringol Cir Cabeza Cuello 2015;75:55-60.

21. van Egmond MMHT, Rovers MM, Tillema AHJ, van Neerbeek N. Septoplasty for nasal obstruction due to a deviated nasal septum in adults: a systematic review. Rhinology 2018;56:195-208.

22. van Egmond MMHT, Rovers MM, Hannink G, Hendriks CTM, van Heerbeek N. Septoplasty with or without concurrent turbinate surgery versus nonsurgical management for nasal obstruction in adults with a deviated septum: a pragmatic, randomised controlled trial. Lancet 2019;394:314-321.

23. Valsamidis K, Titelis K, Rachovitsas D, Konstantinidis I, Markou K, Triaridis S.

Long-Term Evaluation of Nasal Septoplasty Followed by Inferior Turbinate Cauterization for the Treatment of Nasal Obstruction using Objective and Subjective Methods. Int Arch Otorhinolaryngol 2018;22:284-290.

24. Jessen M, Ivarsson A, Malm L. Nasal airway resistance and symptoms after functional septoplasty: comparison of findings at 9 months and 9 years. Clin Otolaryngol Allied Sci 1989;14:231-234.

25. Arunachalam PS, Kitcher E, Gray J, Wilson JA. Nasal septal surgery: evaluation of symptomatic and general health outcomes. Clin Otolaryngol Allied Sci 2001;26:367-370.

26. Gandomi B, Bayat A, Kazemei T. Outcomes of septoplasty in young adults: the Nasal Obstruction Septoplasty Effectiveness study. Am J Otolaryngol 2010;31:189-192.

27. Stewart MG, Smith TL, Weaver EM, Witsell DL, Yueh B, Hannley MT, Johnson JT. Outcomes after nasal septoplasty: results from the Nasal Obstruction Septoplasty Effectiveness (NOSE) study. Otolaryngol Head Neck Surg 2004;130:283-290.

28. Jin HR, Kim DW, Jung HJ. Common Sites, Etiology, and Solutions of Persistent Septal Deviation in Revision Septoplasty. Clin Exp Otorhinolaryngol 2018;11:288-292.

29. Becker SS, Dobratz EJ, Stowell N, Barker D, Park SS, Revision septoplasty: review of sources of persistent nasal obstruction. Am J Rhinol 2008;22:440-444.

30. Floyd EM, Ho S, Patel P, Rosenfeld RM, Gordin E. Systematic Review and Meta-analysis of Studies Evaluating Functional Rhinoplasty Outcomes with the NOSE Score. Otolaryngol Head Neck Surg 2017;156:809-815.

31. Chambers KJ, Horstkotte KA, Shanley K, Lindsay RW. Evaluation of Improvement in Nasal Obstruction Following Nasal Valve Correction in Patients With a History of Failed Septoplasty. JAMA Facial Plast Surg 2015;17:347-350. 


\section{ARTÍCULO DE REVISIÓN}

32. Wang Y, Bonaparte JP. Diagnosis and management of septal deviation and nasal valve collapse - a survey of Canadian otolaryngologists. J Otolaryngol Head Neck Surg 2019;48:71.

33. Ghosh A, Friedman O. Surgical Treatment of Nasal Obstruction in Rhinoplasty. Clin Plast Surg 2016;43:29-40.

34. Apaydin F. Lateral crural turn-in flap in functional rhinoplasty. Arch Facial Plast Surg 2012;14:93-96.

35. Kim L, Papel ID. Spreader Grafts in Functional Rhinoplasty. Facial Plast Surg 2016;32:29-35.

36. Sheen JH. Spreader graft: a method of reconstructing the roof of the middle nasal vault following rhinoplasty. Plast Reconstr Surg 1984;73:230-239.

37. Rohrich RJ, Hollier LH. Use of spreader grafts in the external approach to rhinoplasty. Clin Plast Surg 1996;23:255-262.

38. Wurm J, Kovacevic M. A new classification of spreader flap techniques. Facial Plast Surg 2013;29:506-514.

39. Casanueva F, Astudillo D, Santamaría A, Cardemil F. Autoespaciador en rinoplastía por técnica abierta y cerrada. Rev Otorrinolaringol Cir Cabeza Cuello 2015;75:106-113.

40. Novoa E, Simmen D, Briner HR, Schlegel C. Longterm results after restoring nasal tip support using auricular cartilage as an intercrural columellar strut graft: the "I-Beam" technique. Rhinology 2018;56:183188.

41. Sajjadian A, Guyuron B. An algorithm for treatment of the drooping nose. Aesthet Surg J 2009; 29:199-206.

42. Veit J, Rotter N, Feucht A, Rettinger G, Scheithauer M. Persistent nasal obstruction following septoplasty: deviated nasal pyramid and perpendicular plate. Laryngorhinootologie 2012;91:363-367.

43. Joe SA. The assessment and treatment of nasal obstruction after rhinoplasty. Facial Plast Surg Clin North Am 2004;12:451-458.

44. Rohrich RJ, Deuber MA. Nasal tip refinement in primary rhinoplasty: the cephalic trim cap graft. Aesthet Surg J 2002;22:39-45.

45. Webster RC, Davidson TM, Smith RC. Curved lateral osteotomy for airway protection in rhinoplasty. Arch Otolaryngol 1977;103:454-458. 